\title{
Effect of taurine and caffeine on sleep-wake activity in Drosophila melanogaster
}

This article was published in the following Dove Press journal:

Nature and Science of Sleep

27 September 2010

Number of times this article has been viewed

\author{
Fang Ju Lin' \\ Michael M Pierce' \\ Amita Sehgal ${ }^{2}$ \\ Tianyi Wu' \\ Daniel C Skipper' \\ Radhika Chabba' \\ 'Department of Biology, Coastal \\ Carolina University, Conway, SC, \\ USA; ${ }^{2}$ Department of Neuroscience, \\ $\mathrm{HHMl}$ at University of Pennsylvania, \\ Philadelphia, PA, USA
}

Correspondence: Fang Ju Lin Department of Biology, Coastal Carolina University, P.O. Box 261954, Conway, SC 29528, USA

Tel + I 8433496484

Fax + | 843349220 I

Email flin@coastal.edu

\begin{abstract}
Caffeine and taurine are two major neuromodulators present in large quantities in many popular energy drinks. We investigated their effects on sleep-wake control in constant darkness using the fruit fly Drosophila as a model system. It has been shown that caffeine, as the most widely used psychostimulant, can boost arousal through the dopamine pathway in the mushroom bodies of flies. Taurine is a GABA receptor agonist, which is inhibitory to neuronal firing. We show here that flies receiving a low dose of caffeine $(0.01 \%)$ increase locomotor activity by $25 \%$, and decrease total sleep by $15 \%$. Treatment with taurine at $0.1 \%$ to $1.5 \%$ reduces locomotor activity by $28 \%$ to $86 \%$, and shifts it from diurnal to nocturnal. At $0.75 \%$, taurine also increases total sleep by $50 \%$. Our results show that taurine increases sleep, while caffeine, as previously reported, attenuates sleep. Flies treated with both caffeine and taurine exhibit two differential effects which depend upon the ratio of taurine to caffeine. A high taurine:caffeine ratio promotes sleep, while a low ratio of taurine:caffeine inhibits sleep to a greater extent than the equivalent amount of caffeine alone. This intriguing enhancement of caffeine action by low doses of taurine may account for the presence of both compounds in energy-promoting drinks such as Red Bull ${ }^{\circledR}$ and Monster ${ }^{\circledR}$.
\end{abstract}

Keywords: caffeine (trimethylxanthine), energy drinks, GABA ( $\gamma$-aminobutyric acid), locomotor activity, sleep, taurine (2-aminoethanesulfonic acid)

In the last decade, energy drinks such as Red Bull ${ }^{\circledR}$ and Monster $^{\circledR}$ have become cultural icons and are frequently marketed towards and consumed by young adults. These drinks are intended to keep people awake at work or during leisure activities. Unfortunately, the frequent consumption of these beverages has been linked to risky behavior among college students. ${ }^{1}$ Two common ingredients found in energy drinks are caffeine and taurine, both classified as neuromodulators. The lack of regulation of caffeine content that could lead to caffeine intoxication, and its recreational use in combination with alcohol, have become growing concerns (see review by Reissig). ${ }^{2}$ According to US Food and Drug Administration (FDA) regulations, the maximum caffeine content allowed is $71 \mathrm{mg} / 12 \mathrm{fl} . \mathrm{oz}$. (or $0.02 \%$ ), ${ }^{3}$ yet many energy drinks have clearly exceeded that limit for a single serving without any warning label. The amount of caffeine per serving in energy drinks ranges from $76-80 \mathrm{mg}$ or $0.03 \%$ (eg, original Red Bull ${ }^{\circledR}$ or Monster $^{\circledR}$ ), equivalent to that in a single cup of coffee, to $280 \mathrm{mg}$ or $0.11 \%$ (eg, Cocaine ${ }^{\circledR}$ ) in 8.3 oz. or $250 \mathrm{ml}$. Monster XXL ${ }^{\circledR}$ has as much as $240 \mathrm{mg}$ of caffeine in a $23.5 \mathrm{oz}$. can. The side effects of caffeine include insomnia, nausea, heart palpitations, and even death in rare cases. Caffeine is also known to delay the onset of sleep, shorten stage 3-4 nonrapid eye movement (NREM) sleep and reduce total sleep. ${ }^{4}$ 
In addition, taurine, a sulfhydryl amino acid, found in many tissues including the CNS and muscles, is involved in osmotic regulation ${ }^{5}$ and in modulating the release of other amino acid transmitters at synapses. ${ }^{6}$ Reported benefits of taurine include cytoprotection $^{7}$ and anti-seizure/epilepsy effects. ${ }^{8}$ Both Red Bull $^{\circledR}$ and Monster ${ }^{\circledR}$ have $1000 \mathrm{mg}$ or $0.4 \%$ of taurine in the original $250 \mathrm{ml}$ packages, and the amount triples in Monster $\mathrm{XXL}^{\circledR}$. Though manufacturers claim that caffeine and taurine are safe and beneficial, the interaction between caffeine and taurine has not fully been investigated.

The fruit fly Drosophila melanogaster serves as a useful model to study the sleep-wake cycle since it shares conserved mechanisms with mammals. ${ }^{9-11}$ The circadian ( $\sim 24$ hour) clock that maintains the timing of the sleep-wake cycle in Drosophila resides in the lateral neurons (LNs) of the brain. Within these neurons, a molecular clock maintained by the products of key clock genes: period (per), timeless (tim), Clock $(C l k)$, and cycle $(c y c)$, results in the rhythmic regulation of a neuropeptide, PDF (pigment-dispersing factor). ${ }^{12}$ In turn, PDF promotes arousal during the late night/early morning via PDF-responsive neurons that control downstream motor activity. A second process controlling the sleep-wake cycle, also known as homeostatic control of sleep, determines the amount of sleep rather than the timing of sleep. Results from the Hendricks and Shaw groups have demonstrated that the rest behavior of Drosophila is independent of the circadian clock, and it exhibits similar characteristics to mammalian sleep behavior. ${ }^{13,14}$ Mushroom bodies (MBs), though best known for their function in learning and memory, are also involved in suppressing locomotion, ${ }^{15}$ and promoting sleep ${ }^{16,17}$ at least in part through serotonin signaling. ${ }^{18}$

Neurotransmitters such as dopamine, glutamate, norepinephrine, orexin, and serotonin are involved in promoting wakefulness in mammals, ${ }^{19}$ while adenosine along with other neuropeptides modulate sleep. ${ }^{20,21}$ Similar pathways are present in Drosophila, with arousal controlled by dopamine and octopamine, ${ }^{22,23}$ while sleep is promoted by the GABAergic pathway and the serotonin d5-HT1A receptor. ${ }^{18} \mathrm{GABA}$ elicits a $\mathrm{Cl}^{-}$current and hyperpolarizes the cell membrane, hence it is generally considered to be an inhibitory neurotransmitter in Drosophila, ${ }^{24}$ although excitatory GABA receptors have also been identified in invertebrates. ${ }^{25,26}$ Similarly, it has been shown that taurine is a GABA/glycine receptor agonist, ${ }^{27-29}$ though it requires much higher concentration. Agosto ${ }^{30}$ and Parisky ${ }^{31}$ were the first to link sleep behavior to GABA receptors in Drosophila, which were identified in the lateral neurons (LNs). Our specific aims in this study were to: 1) study the effect of taurine on sleep; and 2) examine the caffeine:taurine interaction involved in sleep-wake cycle control, at the same ratio found in the energy drinks.

\section{Materials and methods}

Caffeine and taurine (Sigma-Aldrich, St. Louis, MO) were initially dissolved in distilled water to make stock solutions and then mixed in sucrose-agar media (5\% sucrose and 1\% agar) to achieve the desired concentrations. Single treatment of caffeine included $0.01 \%, 0.05 \%$, and $0.1 \%$. Taurine at concentrations from $0.125 \%$ to $1.5 \%$ was also used in single treatments. Various nontoxic ranges of taurine/caffeine mixtures were included: 12.5:1 (0.125\% taurine and 0.01\% caffeine), 25:1 (0.25\% taurine and 0.01\% caffeine), 50:1 ( $0.5 \%$ taurine and $0.01 \%$ caffeine), and $75: 1$ (0.75\% taurine and $0.01 \%$ caffeine).

White-eyed $\left(\mathrm{w}^{-}\right)$flies are wild type in circadian behavior and therefore used here for both the control and treatment groups. For the locomotor activity assays, individual glass tubes, each containing a single male fly and sucrose-agar media, were placed in a locomotor monitor (Trikinetics, Waltham, MA). Prior to recording, 5-7-day old flies were entrained to the 12 hour light (10:00 to 22:00): 12 hour dark (22:00 to $10: 00)$ cycle at room temperature $\left(\sim 22^{\circ} \mathrm{C}\right)$, on a Jazz-mix diet (Fisher Scientific, Pittsburgh, PA). All recordings were performed under constant darkness (DD) for 5-9 days (as indicated in figure legends) at $25^{\circ} \mathrm{C}$, to observe endogenous clock activity without environmental cues such as light or sound. In an actogram (as seen in Figure 1B), the number on top of the $\mathrm{x}$ axis represents a 24-hour period of recordings, from midnight $(00: 00 \mathrm{~h})$ to $00: 00 \mathrm{~h}$ of the next day. The number on the y axis from top to bottom indicates successive days of continuous recordings, with each line representing a 24 hour period. Locomotor activity was measured by recording the number of times the infrared light equipped within each locomotor monitor was interrupted due to the movement of a fly. The total number of movements were summed at the end of each 30-minute interval, and shown as a single bar on each line. The height of the bar is proportional to the number of movements recorded in a 30-minute period. The activity from all groups in the same Figure was adjusted to the same scale (indicated on the upper right-hand corner of each actogram). All data were collected with DAMSystem Collection software (Trikinetics) and analyzed using ClockLab software (Actimetrics, Wilmette, IL).

To measure sleep behavior, flies were entrained and treated the same way as described above, except that the locomotor activity was collected at 5-minute intervals. A sleep bout is 
defined as no locomotor activity observed within a 5-minute period. ${ }^{32}$ Sleep bouts measured over a 5-7-day recording period were expressed either as total minutes of sleep per 24 hours (total sleep), or minutes per hour (for subjective day or subjective night). All recordings were obtained in constant darkness for 5-7 days in an incubator set to $25^{\circ} \mathrm{C}$. Statistical analysis was performed using a Wilcoxon Rank
Sum Test (two independent sample) from SOCR (Statistics Online Computational Resource; http://nsdl.org/resource/2 200/20061003060017201T). Locomotor activity or sleep in caffeine- or taurine-only treatments was compared to sham control, whereas locomotor activity or sleep in the taurine: caffeine combined group was compared to that of the equivalent dose of taurine and caffeine separately.

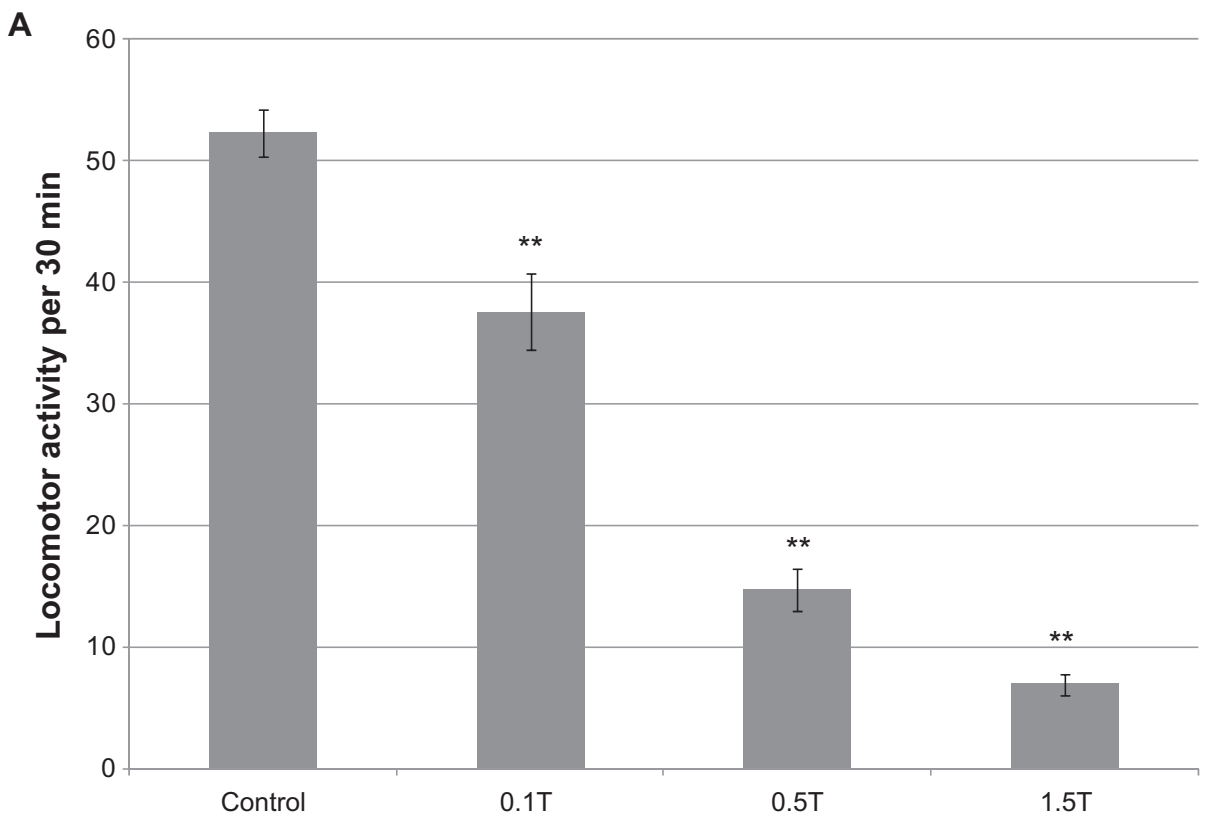

B
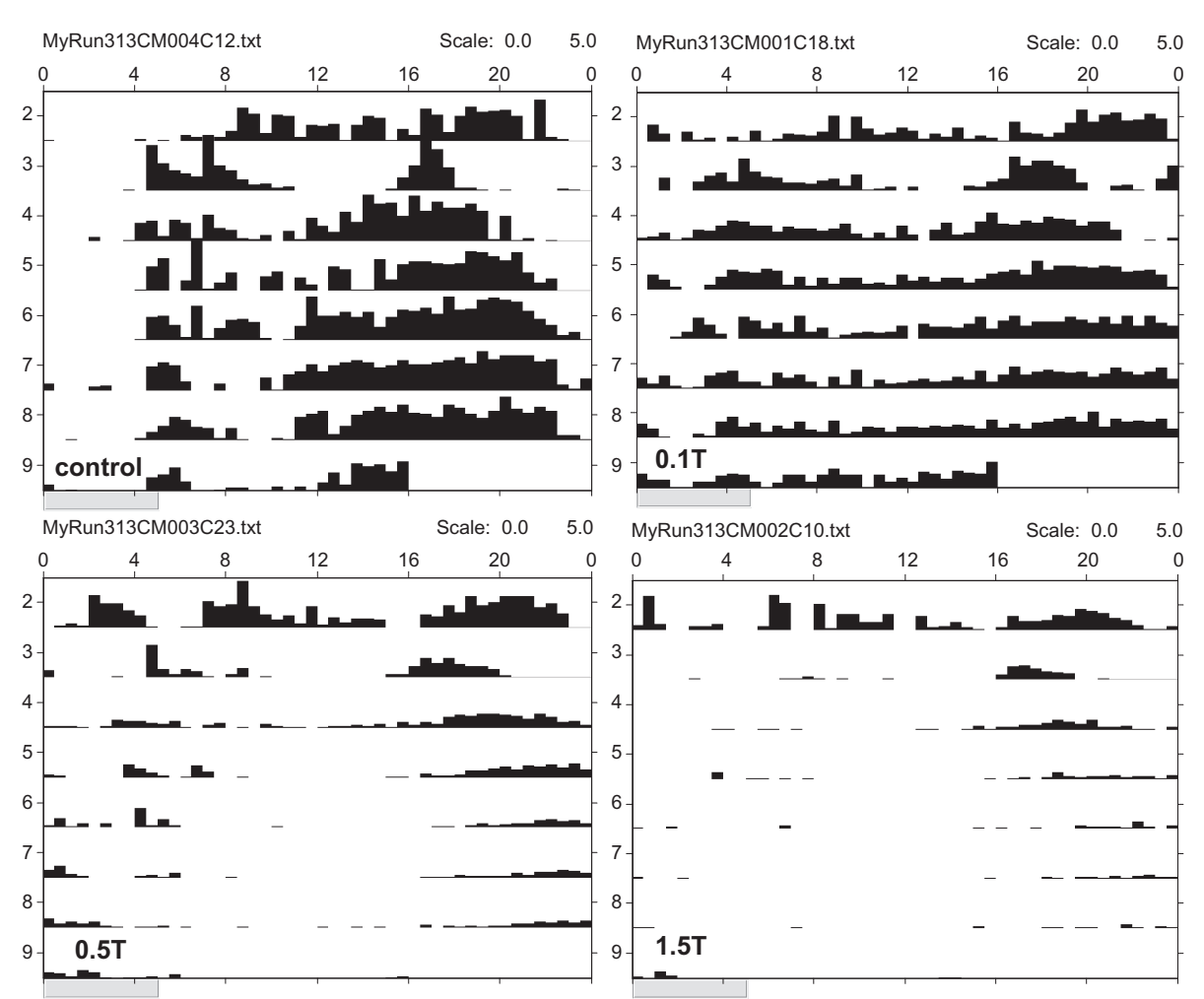

Figure I (Continued) 

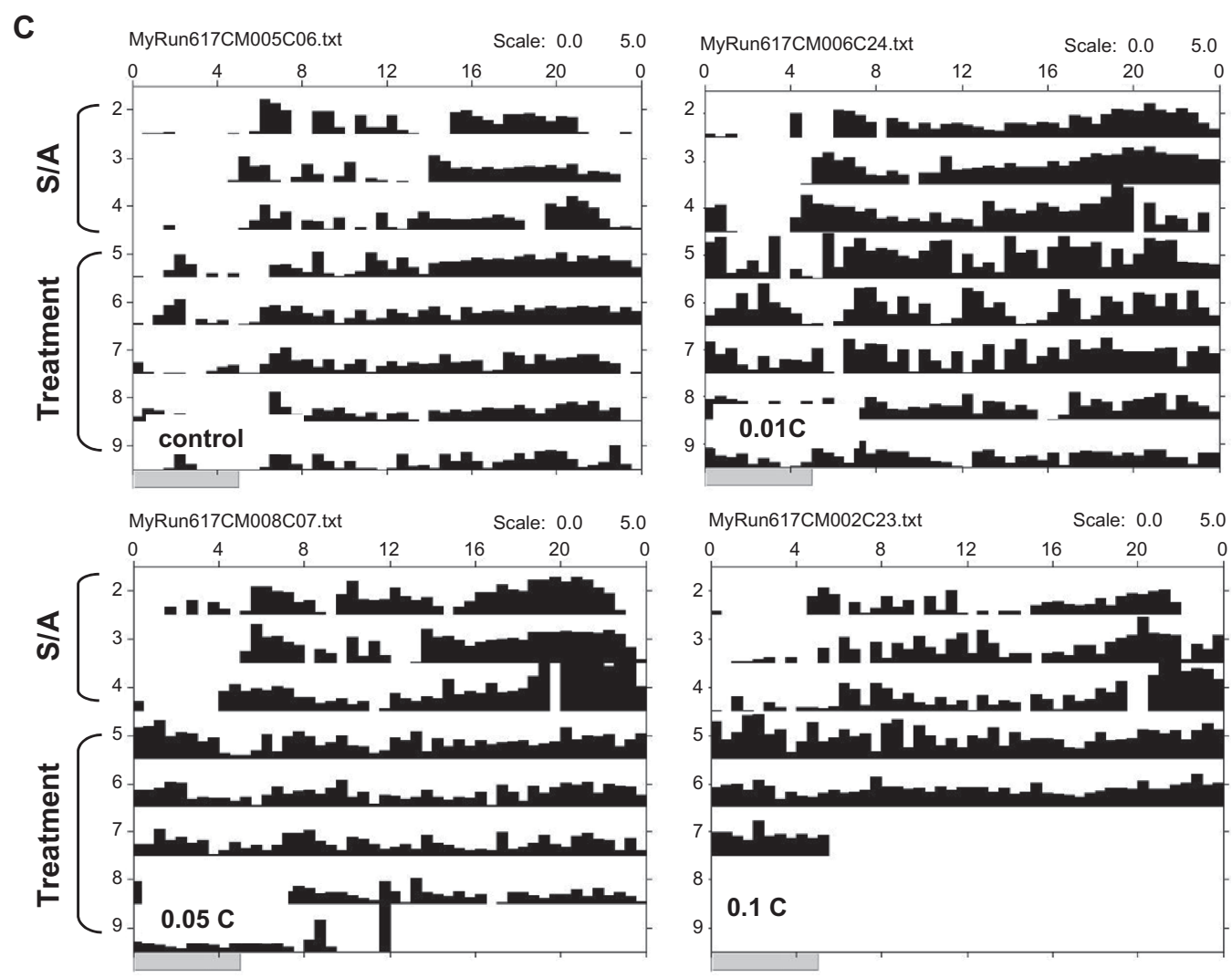

Figure I Locomotor activity in taurine- $(\mathrm{T})$ and caffeine- $(\mathrm{C})$ treated flies. $\mathrm{A}$ ) Taurine treatment decreases locomotor activity. Data shown are from control $(\mathrm{n}=22), 0.1 \% \mathrm{~T}$ $(n=19), 0.5 \% T(n=12)$, and I.5\%T $(n=10)$. Average movements in a 30 minute window were calculated over 5 days, and indicated on the $y$ axis. Value represents mean \pm standard error of the mean (SEM). Asterisks (* or **) indicate $P<0.05$ and $P<0.005$ compared to the control, respectively. B) Representative activity record (actogram) of a single fly recording from each treatment group: control, $0.1 \% \mathrm{~T}, 0.5 \% \mathrm{~T}$, and $1.5 \% \mathrm{~T}$. The $\mathrm{x}$ axis indicates a 24 hour timeline on top of each actogram. The shaded areas represent activity which is measured as described in Methods. Activity profiles from day 2 to day 9 are shown (y axis). The amplitude of activity is adjusted to the same scale (from 0-5) in each activity record. C) Caffeine increases locomotor activity at higher concentrations. Shown here is representative activity record of a single fly recording from each treatment group (control, $0.01 \% \mathrm{C}, 0.05 \% \mathrm{C}$, and $0.1 \% \mathrm{C}$ ), with sucrose/agar media (S/A) in the first three days (day 2 to 4 on $y$ axis) then transferred to either S/A only (eg, control), or sucrose/agar with various concentrations of caffeine (days 4-9). The arrow indicates time of transfer on day 4.

\section{Results}

\section{Effect on locomotor activity by taurine or caffeine alone}

The effects of taurine or caffeine on Drosophila locomotor activity were tested by recording the movement of flies over a 24-hour period. The locomotor activity of flies was decreased by $28 \%$ to $86 \%$ with increasing taurine concentration (Figure 1 A). Interestingly, while the daytime activity quickly diminished, the residual locomotor activity was shifted to the nighttime (Figure 1B). This effect was more pronounced in flies that received higher doses of taurine. In contrast, caffeine increased both the frequency as well as the amplitude of locomotor activity. For the $0.01 \%$ caffeine-treated group, flies had sustained high activity without lethality. With higher doses of caffeine $(0.05 \%$ and $0.1 \%)$, there were initial bursts of high activity but most flies subsequently died (Figure 1C). Five-day survival rates were $96 \%, 97 \%, 72 \%$, and $57 \%$, for sham control and various doses of caffeine $(0.01 \%, 0.05 \%$, $0.1 \%$ ) respectively (data not shown).

\section{Inhibition of locomotor activity by taurine is reversible}

To determine whether the inactivity in the taurine-treated groups was the result of permanent damage of neuromuscular function, flies were first treated with $1.5 \%$ taurine for 5 days, and then transferred to regular media lacking taurine; locomotor activity was recorded for three additional days. The locomotor activity of flies that were exposed to taurine first and switched to normal media was similar to that of the control flies $(P=0.908)$, and eight fold higher than that of flies that remained in taurine (Figure 2). Thus, the inhibition of locomotion by taurine is reversible. Diurnal activity also emerged rapidly in the reversal group, supporting the notion that taurine alters the circadian component that controls daytime activity.

\section{Effect of taurine: caffeine mixture on locomotor activity}

The combined effect of taurine and caffeine on locomotion (Figure 3) and sleep (Figure 4) was measured using mixtures 
A

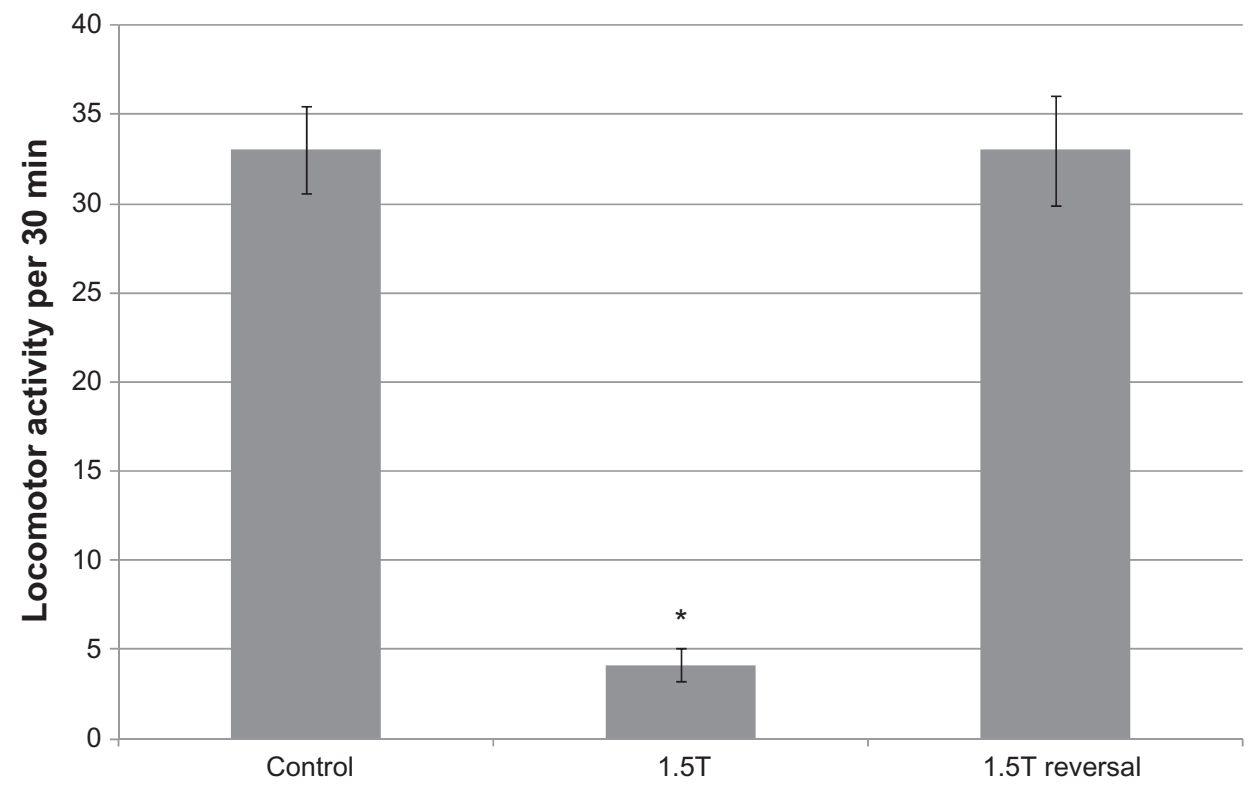

B

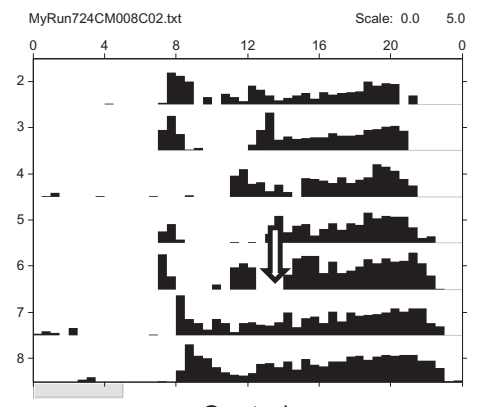

Control

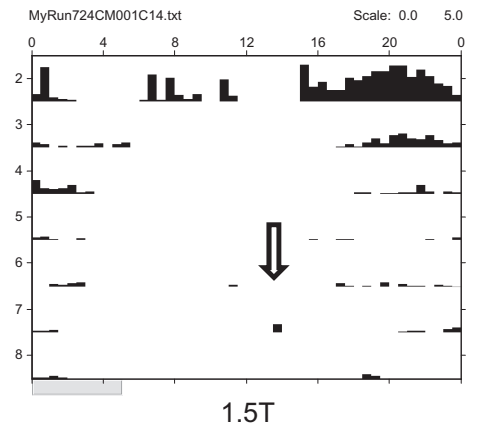

$1.5 \mathrm{~T}$

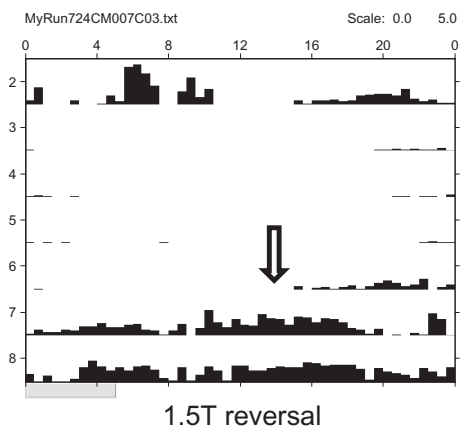

1.5T reversal

Figure 2 Effect of taurine on locomotion is reversible. Locomotor activity of the sham control and taurine-treated flies (I.5T and I.5T reversal) were monitored for 5 days. On day 6, flies were transferred to fresh tubes containing either sucrose/agar media (control and I.5 reversal groups), or I.5\% taurine (I.5T), and returned to the monitor for a further four days. A) Data shown are average of 4-day locomotor activity after switching. Values represent mean \pm S.E.M. from each group ( $\mathrm{n}=32$ in control, and $\mathrm{n}=16$ in both I.5T and I.5T reversal groups). No significant difference was observed between control and the I.5T reversal groups. B) Representative activity records of individual flies. The arrows indicate the time of transfer on day 6.

of a constant concentration of caffeine $(0.01 \%)$ and increasing concentrations of taurine from $0.125 \%$ to $0.75 \%$. The lowest ratio of taurine to caffeine used in our experiment is identical to the ratio present in the Red Bull ${ }^{\circledast}$ energy drink. None of the caffeine, taurine, or combined concentrations used were lethal to flies. Both locomotor activity and sleep were recorded for all mixtures of taurine and caffeine. Flies receiving the taurine-only treatment showed a dose-dependent decrease in locomotor activity, though only the $0.5 \%$ and $0.75 \%$ concentrations showed a significant difference from the control (Figure 3A; $P<0.005$ ). Flies treated with $0.01 \%$ caffeine showed an approximately $30 \%$ increase in locomotor activity. At the lowest ratio of taurine to caffeine (12.5:1), flies exhibited a similar level of locomotor activity to either the caffeine-only $(P=0.41)$ or taurine-only group $(P=0.1)$. In groups with higher taurine:caffeine ratios locomotor activity was lower than that of the equivalent dose of caffeine alone $(P<0.01)$, but not different from that of the equivalent dose of taurine alone. These results suggest that the inhibitory effect of taurine quenches the stimulatory effect of caffeine as the level of taurine is increased in the mixture.

\section{Effect of taurine: caffeine mixture on sleep behavior}

We further investigated how caffeine and taurine either individually or in combination can impact sleep. For Drosophila, a sleep bout is defined as 5 minutes of inactivity. ${ }^{8}$ As expected, caffeine reduced the amount of sleep $(\sim 18 \%)$, whereas the highest dose of taurine $(0.75 \%)$ increased total sleep by approximately $50 \%$ when compared to the control $(P<0.005$, 
A

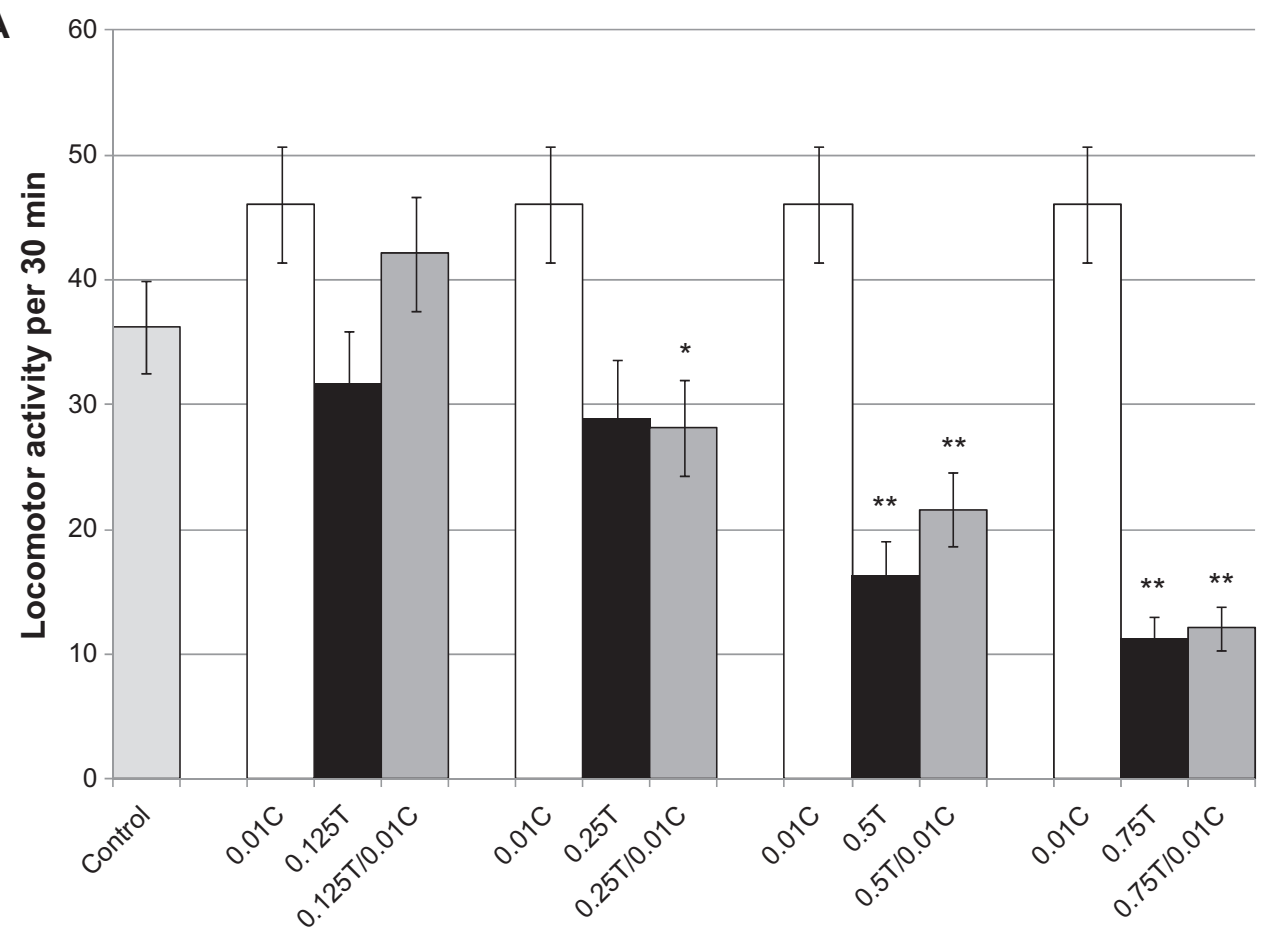

B

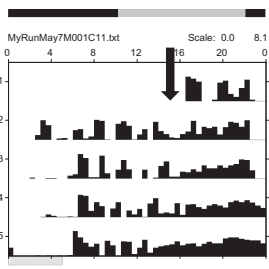

Control

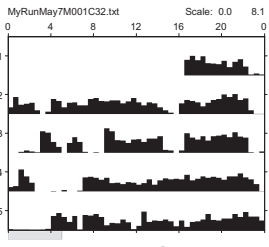

$0.01 \mathrm{C}$

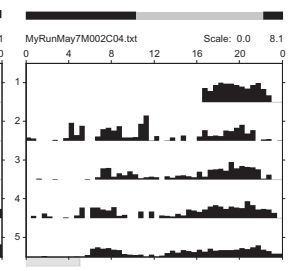

$0.125 \mathrm{~T}$

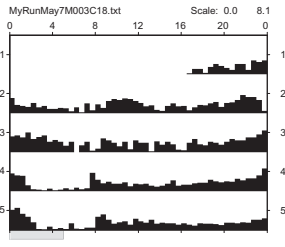

$0.01 \mathrm{C} / 0.125 \mathrm{~T}$

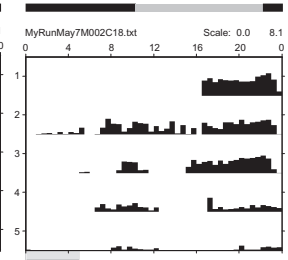

$0.25 \mathrm{~T}$

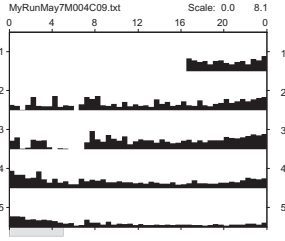

$0.01 \mathrm{C} / 0.25 \mathrm{~T}$

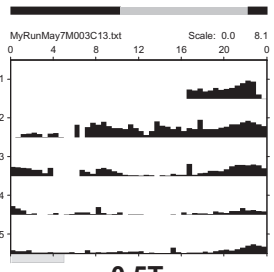

$0.5 \mathrm{~T}$

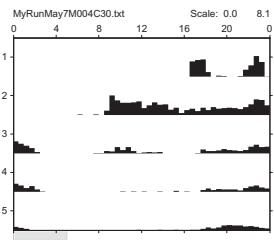

$0.01 \mathrm{C} / 0.5 \mathrm{~T}$

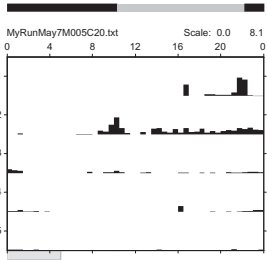

$0.75 \mathrm{~T}$

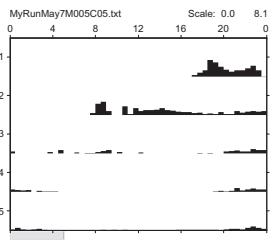

$0.01 \mathrm{C} / 0.75 \mathrm{~T}$

Figure 3 Taurine alone or in combination with caffeine attenuates locomotor activity. A) Average locomotor activity from flies treated with sucrose/agar (control), $0.01 \%$ caffeine $(0.0 \mathrm{IC})$ alone, or $0.0 \mathrm{IC}$ with taurine ranging from $0.125 \%$ to $0.75 \%(0.125 \mathrm{~T}, 0.25 \mathrm{~T}, 0.5 \mathrm{~T}$, and $0.75 \mathrm{~T})$ with between 12 and 16 flies in each group. Asterisks (* or $* *)$ indicate $P$ values that are smaller than 0.05 and 0.005 , respectively. B) Representative activity records of flies from groups in (A). The arrow indicates the beginning of recording on day I. The shaded rectangle at the top of the actogram indicates subjective day (10:00 to 22:00) and solid dark rectangle indicates subjective night (22:00 to I0:00).

Figure 4 A). Surprisingly, at the lowest taurine:caffeine ratio (12.5:1), the amount of total sleep observed was less than that observed in the presence of caffeine $(P<0.05)$ or taurine alone $(P<0.005)$. At higher taurine:caffeine ratios, the amount of sleep is similar to that of equivalent dose of taurine but higher than that in the presence of caffeine $(P<0.05$ and $P<0.005$ for 50:1 and 75:1, respectively). Total sleep was subsequently broken down into subjective daytime (10:00 to 22:00) and subjective nighttime (22:00 to 10:00) sleep (Figure 4B). Daytime or nighttime sleep was increased in all groups containing taurine concentrations of $0.5 \%$ or higher. At the lowest ratio
(12.5:1), daytime sleep was less than that observed for taurine $(P<0.005)$ or caffeine treated flies $(P<0.05)$, while nighttime sleep was similar to that produced by $0.01 \mathrm{C}(P=0.244)$ but different from that in taurine-treated flies $(P<0.05)$. Taken together, taurine not only decreases activity and increases sleep, but also appears to induce a greater change in daytime sleep, compared to that of nighttime sleep.

\section{Discussion}

This study is the first in which the effect of taurine on both locomotor activity and sleep is reported. Our results demonstrate 
A

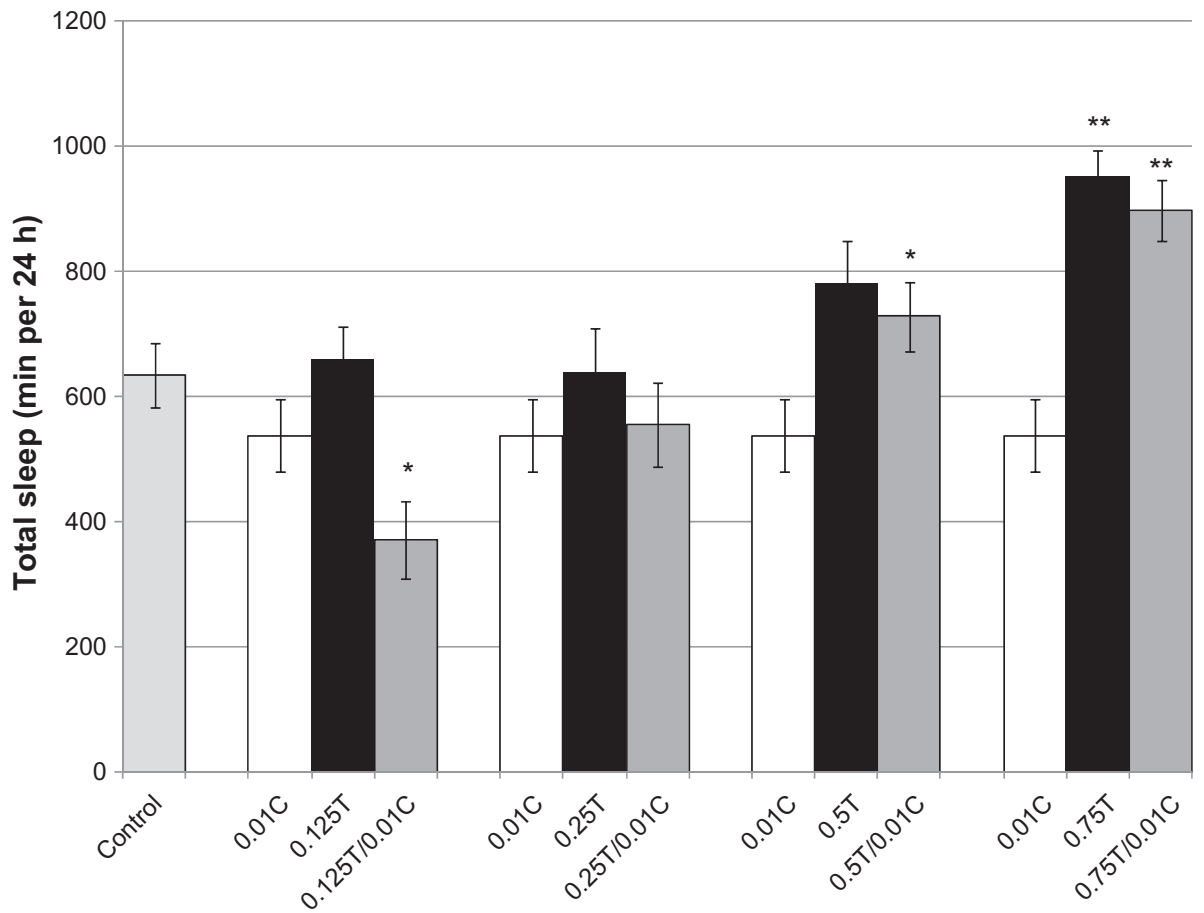

B

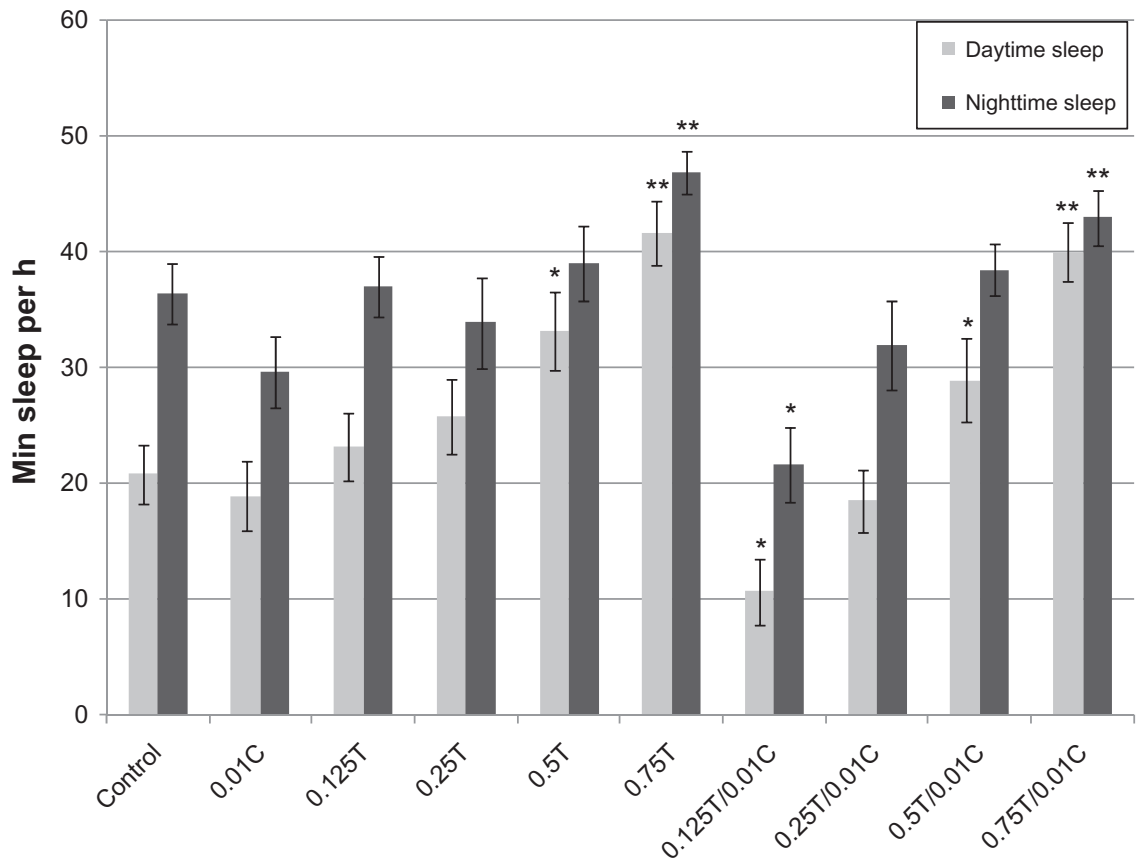

Figure 4 Taurine and caffeine have opposite effects on sleep. A) Total sleep is shown as minutes of sleep in a 24 hour period. Between 12 and 16 flies were used in each group. B) Number of sleep bouts from nighttime sleep (22:00 to 10:00) or daytime sleep (10:00 to 22:00) are collected from three consecutive days or nights (day 2-4), and converted to minutes of sleep per hour. All caffeine-only or taurine-only groups were compared to the control, whereas the taurine/caffeine combined groups were compared to corresponding caffeine or taurine only treatments at the same concentration.

that: 1) taurine reduces locomotor activity in a dose-dependent manner; 2) while caffeine alone at $0.01 \%$ increases locomotor activity, the addition of taurine attenuates the effect of caffeine when the taurine:caffeine ratio reaches $25: 1$ or higher; and 3) at the lowest taurine to caffeine ratio $(12.5: 1)$, total sleep is reduced and fragmented to a greater extent than by caffeine alone.
In Drosophila, rhythmic locomotor activity is under the control of several clock protein-expressing neurons, which send projections to the accessory medulla, the medulla, and the dorsal protocerebrum. These structures then interact with other regions of the brain through either electrical or humoral pathways. ${ }^{9}$ The rhythmic expression of PDF is important not 
only for arousal in the daytime, but also for regulating sleep through the interaction with other neurons. For example, a $p d f$ mutant fly is arrhythmic under conditions of constant darkness ${ }^{12}$ and also shows increased sleep. ${ }^{33}$ Other regions of the Drosophila brain, such as the mushroom bodies, are involved in the homeostatic regulation of sleep. ${ }^{16,17}$ The Drosophila dopamine receptor, $\mathrm{dDA} 1,{ }^{34}$ has been shown to be expressed in MBs and to mediate caffeine-induced arousal. ${ }^{35} \mathrm{Wu}$ and colleagues further demonstrated that caffeine activates the cAMP-PKA pathway, resulting in increased cAMP levels and fragmented sleep in both wild type flies and in flies lacking a functional adenosine receptor (AdoR1). ${ }^{36}$ Though adenosine receptors are important for mediating caffeine-induced arousal in mammals, it does not appear to be true for Drosophila. Our result supports the previously published data that caffeine treatment alone increases locomotor activity, as well as decreases total sleep.

As mentioned in the Introduction, taurine, a GABA receptor agonist, is the second most abundant amino acid in the mammalian CNS and is also normally present in MBs in Drosophila. ${ }^{37}$ Taurine can be released from either glia or neurons during stress conditions. ${ }^{38}$ Jia reported that taurine concentrations in the range of $10-100 \mu \mathrm{M}$ activate $\mathrm{GABA}_{\mathrm{A}}$ receptors in the mouse thalamus, which plays an important role in the regulation of sleep and wakefulness. ${ }^{39}$ Additionally, Agosto et al reported shortened latency to sleep onset and increased sleep in flies carrying a mutated $\mathrm{GABA}_{\mathrm{A}}$ receptor, Rdl (resistance to dieldrin), which has a longer period of channel opening. ${ }^{30}$ Parisky et al demonstrated that overexpression of Rdl, a GABA subunit found in ventrolateral neurons (LNvs), increases total sleep, while reducing Rdl level decreases sleep. ${ }^{31}$ Another GABA receptor, $\mathrm{GABA}_{\mathrm{B}}$, was also found in pdf-positive neurons, ${ }^{40}$ further supporting the notion that GABA and ventrolateral neurons modulate sleep circuitry in Drosophila. Our data support our hypothesis that taurine, as a GABA receptor agonist, promotes sleep. Based on the presence of GABA receptors, we propose that taurine may target: A) ventrolateral neurons, resulting in lower locomotor activity and more sleep; B) neurons that mediate caffeine-induced arousal. A possible explanation for the switch from diurnal to nocturnal locomotor activity is that once homeostatic need is met during the day, locomotor activity resumes in the nighttime. A similar observation was previously reported by Crocker and Sehgal. ${ }^{23}$ In their study, a mutation in $\mathrm{T} \beta \mathrm{H}$ (tyramine beta hydroxylase) which is involved in octopamine biosynthesis resulted in a reduced level of octopamine and an accumulation of its precursor tyramine. $\mathrm{T} \beta \mathrm{H}$ mutant flies exhibit increased daytime sleep, but also show more locomotor activity in the night.

In this study, biphasic responses in sleep behavior were observed when taurine and caffeine were combined. At the lowest taurine to caffeine ratio, flies exhibit similar locomotor activity (Figure 3A) but decreased sleep, as compared to flies treated with caffeine alone (Figure 4A). The lowest ratio of taurine:caffeine also results in less consolidated sleep than taurine or caffeine alone (supplemental Figure S1). It is possible that at this low concentration taurine shifts the distribution of locomotor activity (changing it to more nighttime hours) that offsets from caffeine's locomotor rhythms, resulting in less sleep than that of caffeine alone (supplemental Figure S2). Thus, episodes of sleep which are 16 minutes or higher are fragmented with this low ratio of taurine:caffeine combination. The same ratio of taurine to caffeine $(12.5: 1)$ present in Red Bull produces the desired effect of energy drinks: sustained activity that is enhanced compared to beverages that contain only caffeine but not taurine. However, this effect is reversed with a higher taurine:caffeine ratio, indicating that a higher concentration of taurine is capable of inhibiting the caffeine effect completely.

It is possible that taurine inhibits mobility by directly activating GABA receptor at the neuromuscular junction. ${ }^{41}$ Leal and Neckameyer ${ }^{42}$ have shown that millimolar amounts of GABA $(0.1 \%-0.5 \%$; equivalent to $9-48 \mathrm{mM})$ can decrease locomotor activity in Drosophila. To elicit a similar response of the GABA receptor by taurine, it usually requires a 1000-fold higher concentration. ${ }^{5}$ The taurine concentrations used in this study were much lower than required for the direct inhibition of mobility. Additionally, if the neuromuscular system, rather than CNS, is the sole target of taurine, we would have expected to see low or no activity throughout the 24 hour recording period. Instead, we observed that low nighttime activity persisted even at the highest concentrations of taurine (Figure 1). Our results also indicate that the inactivity observed in taurine-treated flies is not due to permanent damage to muscles or to the neuromuscular junctions (Figure 2). Furthermore, to address whether the inactivity was simply due to a fly's aversion to the smell or taste of taurine, fruit flies were placed in tubes containing either regular media, or media with $1.5 \%$ taurine. After a 2-hour incubation period, flies were transferred to tubes containing only moist cotton and locomotor activity was recorded for another 24 hours. In the absence of the cue (ie, smell or taste) from food, the activity of the taurine-treated group was still 50\% lower than that of the sham control (data not shown). Although we cannot rule out the direct inhibition of the neuromuscular system 
by taurine, our data suggest that the mechanism of action is likely through the inhibition of the CNS.

Overall our study results demonstrate that the combination of caffeine and taurine at the same ratio as in energy drinks has synergistic effects in shortening sleep and can lead to sleep deprivation and subsequent attention deficit in the long run. However, sleep-wake behavior is unlikely the only target of caffeine and taurine. For example, it has been shown that taurine alone protects against cardiac injury by preventing Ca overload (see review by $\mathrm{Xu}^{43}$ ). The presence of taurine in energy drinks may alleviate or even quench the uncomfortable side effects of caffeine, such as heart palpitation, jitteriness, and anxiety. Kong's group has previously demonstrated an anxiolytic-effect of taurine in a rat anxiety model. ${ }^{44}$ The propensity of taurine to blunt the symptoms of caffeine intoxication, raises important health concerns. In Europe, Red Bull ${ }^{\circledR}$ was banned after a few cases of cardiac arrest occurred due to ingestion of large amounts of energy drinks. Consumers would presumably stop drinking caffeine-containing beverages if they experienced heart discomfort, nausea, or twitching muscles, but these symptoms are likely alleviated by taurine. Thus, the threshold for perceiving a caffeine overdose is raised with taurine in the drink. Second, taurine interacts with the GABA receptor, which is a key component for maintaining neuronal plasticity. In the case of long term consumption of energy drinks, high doses of taurine can accumulate in tissues through the taurine transporter and be released during stress. ${ }^{38,45}$ Higher than normal levels of taurine at synapses could interact with GABA receptors, cause receptor desensitization and/or alteration of GABA homeostasis, and lead to neurological disorders. Along these lines, Iyadurai and Chung reported a possible link between energy consumption and new-onset seizure in four patients. ${ }^{46}$ With the majority of energy drink consumers being children or adolescents, clear labeling of caffeine content and its side effects, as well as age limitations should be mandated and enforced. Interactions among other added ingredients should also be addressed; indeed, a synergistic effect of sugar and caffeine was observed on cognitive performance. ${ }^{47}$ Although in the present study we only focused on the interaction of caffeine and taurine on locomotion and sleep, other analyses such as social behaviors or toxicity of overloaded supplements are required to assess the potential long-term impact of energy drink consumption in humans.

\section{Acknowledgments}

The authors thank Stormi Chadwick and Greer Baxter for their assistance in measuring fly locomotor activity; Dr. Karen Aguirre and Dr. Michael Roberts from Coastal Carolina University for helpful discussions and for reviewing the manuscript; Dr. Kevin Godwin from Coastal Carolina University for his expertise in statistical analyses; and Molly French for her technical assistance in the preparation of the Figures.

\section{Disclosure}

The authors report no conflicts of interest in this work.

\section{References}

1. Miller KE. Energy drinks, race, and problem behaviors among college students. J Adolesc Health. 2008;43(5):490-497.

2. Reissig CJ, Strain EC, Griffiths RR. Caffeinated energy drinks - a growing problem. Drug Alcohol Depend. 2009;99(1-3):1-10.

3. Food and Drug Administration. Substances generally recognized as safe. Code of Federal Regulations. 2003; Title 21 volume 2, Sec 182.1180 Accessed at: http://www.cfsan.fda.gov/ 1rd/fcf182.html

4. Roehrs T, Roth T. Caffeine: sleep and daytime sleepiness. Sleep Med Rev. 2008;12:153-162.

5. Huxtable RJ. Physiological actions of taurine. Physiol Rev. 1992;72(1): $101-163$.

6. Foos TM, Wu JY. The role of taurine in the central nervous system and the modulation of intracellular calcium homeostasis. Neurochem Res. 2002;27:21-26.

7. Ricci L, Valoti M, Sgaragli G, et al. Protection by taurine of rat brain cortical slices against oxygen glucose deprivation- and reoxygenationinduced damage. Eur J Pharmacol. 2009;621(1-3):26-32.

8. Gaby AR. Natural approaches to epilepsy. Altern Med Rev. 2007;12(1):9-24.

9. Helfrich-Forster C. Neurobiology of the fruit fly's circadian clock. Genes Brain Behavior. 2005;4:65-76.

10. Rosenwasser AM. Functional neuroanatomy of sleep and circadian rhythms. Brain Res Rev. 2009;61:281-306.

11. Sheeba V. The Drosophila melanogaster circadian pacemaker circuit. J Genet. 2008;87:485-493.

12. Renn SCP, Park JH, Rosbash M, Hall JC, Taghert PH. A pdf neuropeptide gene mutation and ablation of PDF neurons each cause severe abnormalities of behavioral circadian rhythms in Drosophila. Cell. 1999;99:791-802.

13. Hendricks JC, Finn SM, Panckeri KA, et al. Rest in Drosophila is a sleep-like state. Neuron. 2000;25:129-138.

14. Shaw PJ, Cirelli C, Greenspan RJ, Tononi G. Correlates of sleep and waking in Drosophila melanogaster. Science. 2000;287:1834-1837.

15. Martin JR, Ernst R, Heisenberg M. Mushroom bodies suppress locomotor activity in Drosophila melanogaster. Learn Mem. 1998;5:179-191.

16. Pitman JL, McGill JJ, Keegan KP, Allada R. A dynamic role for the mushroom bodies in promoting sleep in Drosophila Nature. 2006;441: 753-756.

17. Joiner WJ, Crocker A, White BH, Sehgal A. Sleep in Drosophila is regulated by adult mushroom bodies. Nature. 2006;441:757-760.

18. Yuan Q, Joiner WJ, Sehgal A. A sleep-promoting role for the Drosophila serotonin receptor 1A. Curr Biol. 2006;16(11):1051-1062.

19. Miller DB, O'Callaghan JP. The pharmacology of wakefulness. Metabolism. 2006;55:S13-S19.

20. Landolt HP. Sleep homeostasis: a role for adenosine in humans? Biochem Pharmacol. 2008;75:2070-2079.

21. Sakurai T. Roles of orexin/hypocretin in regulation of sleep/wakefulness and energy homeostasis. Sleep Med Rev. 2005;9:231-241. 
22. Kume K, Kume S, Park SK, Hirsh J, Jackson FR. Dopamine is a regulator of arousal in the fruit fly. J Neurosci. 2005;25(32):7377-7384.

23. Crocker A, Sehgal A. Octopamine regulates sleep in Drosophila through PKA dependent mechanisms. J Neurosci. 2008;28(38):9377-9385.

24. Lee D, Su H, O’Dowd DK. GABA receptors containing Rdl subunits mediate fast inhibitory synaptic transmission in Drosophila neurons. J Neurosci. 2003;23(11):4625-4634.

25. Beg AA, Jorgensen EM. EXP-1 is an excitatory GABA-gated cation channel. Nat Neurosci. 2003;6(11):1145-1152.

26. Gisselmann G, Plonka J, Pusch H, Hatt H. Drosophila melanogaster GRD and LCCH3 subunits form heteromultimeric GABA-gated cation channels. Br J Pharmacol. 2004;142:409-413.

27. Ochoa-de la Paz LD, Martinez-Davila IA, Miledi R, Martinez-Torres A. Modulation of human GABA1 receptors by taurine. Neurosci Res. 2008;61:302-308.

28. Schmieden V, Kuhse J, Betz H. Agonist pharmacology of neonatal and adult glycine receptor subunits: identification of amino acid residues involved in taurine activation. EMBO J. 1992;11:2025-2032.

29. Sergeeva OA, Haas HL. Expression and function of glycine receptors in striatal cholinergic interneurons from rat and mouse. Neurosci. 2001;104:1043-1055.

30. Agosto J, Choi JC, Parisky KM, Stilwell G, Rosbash M, Griffith LC. Modulation of $\mathrm{GABA}_{\mathrm{A}}$ receptor desensitization uncouples sleep onset and maintenance in Drosophila. Nat Neurosci. 2008;11:354-359.

31. Parisky KM, Agosto J, Pulver SR, et al. PDF cells are a GABAresponsive wake-promoting component of the Drosophila sleep circuit. Neuron. 2008;60:672-682.

32. Andretic R, Shaw PJ. Essentials of sleep recordings in Drosophila: moving beyond sleep time. Methods Enzymol. 2005;393:759-772.

33. Chung BY, Kilman VL, Keath JR, et al. The GABA(A) receptor RDL acts in peptidergic PDF neurons to promote sleep in Drosophila. Curr Biol. 2009;19(5):386-390.

34. Kim YC, Lee HG, Han KA. D1 dopamine receptor dDA1 is required in the mushroom body neurons for aversive and appetitive learning in Drosophila. J Neurosci. 2007;27:7640-7647.
35. Andretic R, Kim YC, Jones FS, Han KA, Greenspan RJ. Drosophila D1 dopamine receptor mediates caffeine-induced arousal. PNAS. 2008;105(51):20392-20397.

36. Wu MN, Ho K, Crocker A, Yue Z, Koh K, Sehgal A. The effects of caffeine on sleep in Drosophila require PKA activity, but not the adenosine receptor. J Neurosci. 2009;29(35):11029-11037.

37. Strausfeld NJ, Sinakevitch I, Vilinsky I. The mushroom bodies of Drosophila melanogaster: an immunocytological and Golgi study of Kenyon cell organization in the calyces and lobes. Microsc Res Tech. 2003;62(2):151-169.

38. Oja SS, Saransaai P. Modulation of taurine release by glutamate receptors and nitric oxide. Prog Neurobiol. 2000;62:407-425.

39. Jia F, Yue M, Chandra D, et al. Taurine is a potent activator of extrasynaptic GABA $_{\mathrm{A}}$ receptors in the thalamus. $J$ Neurosci. 2008;28(1):106-115.

40. Hamasaka Y, Wegener C, Nassel DR. GABA modulates Drosophila circadian clock neurons via $\mathrm{GABA}_{\mathrm{B}}$ receptors and decreases in calcium. J Neurobiol. 2005;65:225-240.

41. Dudel J, Adelsberger H, Heckmann M. Neuromuscular glutamatergic and GABAergic channels. Invertebr Neurosci. 1997;3:89-92.

42. Leal SM, Neckameyer WS. Pharmacological evidence for GABAergic regulation of specific behaviors in Drosophila melanogaster. J Neurobiol. 2002;50:245-261.

43. Xu YJ, Arneja AS, Tappia PS, et al. The potential health benefits of taurine in cardiovascular disease. Exp Clin Cardiol. 2008;13(2):57-65.

44. Kong WX, Chen SW, Li YL, et al. Effects of taurine on rat behaviors in three anxiety models. Pharmacol Biochem Behav. 2006;83:271-276.

45. Janaky R, Shaw CA, Oja SS, et al. Taurine release in developing mouse hippocampus is modulated by glutathione and glutathione derivatives. Amino Acids. 2008;34(1):75.

46. Iyadurai SJP, Chung SS. New-onset seizures in adults: possible association with consumption of popular energy drinks. Epilepsy Behav. 2007;10(3):504-508.

47. Adan A, Serra-Grabulosa JM. Effects of caffeine and glucose, alone and combined, on cognitive performance. Hum Psychopharmacol. 2010;25(4):310-317. 


\section{Supplemental figures}

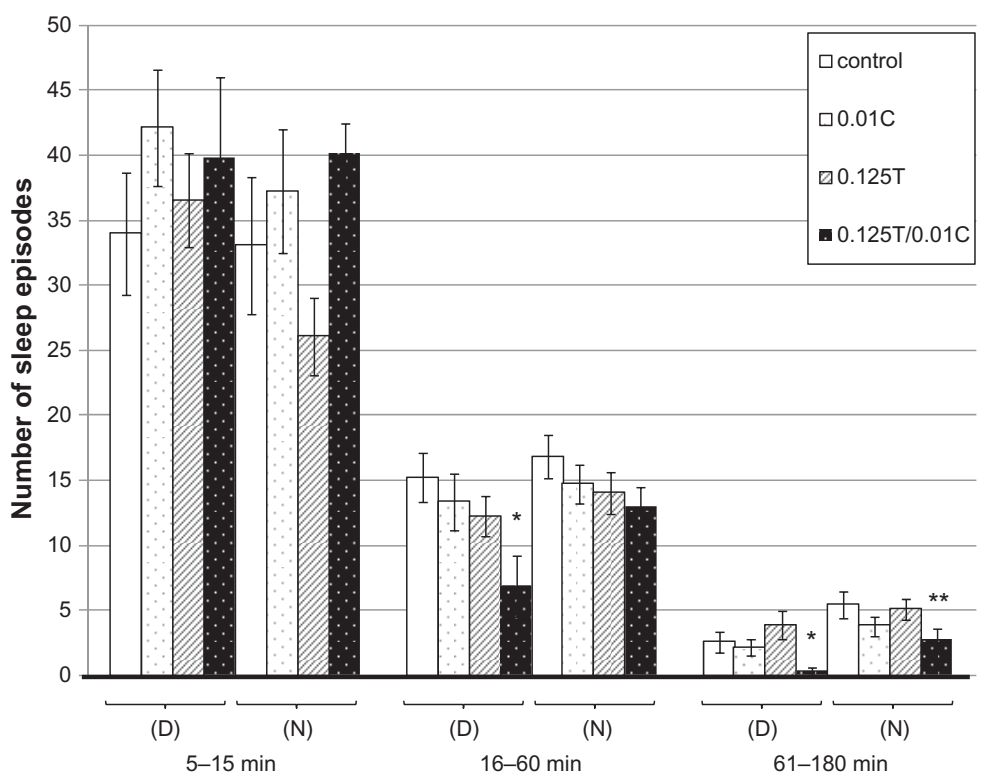

Figure SI Number of sleep episodes were counted during the subjective day $(\mathrm{D})$ or subjective night $(\mathrm{N})$ over four consecutive days, and further divided into three groups: 5-15 minutes, 16-60 minutes, and 6I-180 minutes. The number of sleep episodes observed in $0.125 \mathrm{~T} / 0.0 \mathrm{IC}$-treated flies is lower than that of the $0.01 \mathrm{C}$ - or $0.125 \mathrm{~T}$ treatedflies alone ( $P<0.05$ for both) at the I6-60 minute range (D), and it is also lower than that of $0.125 \mathrm{~T}(P=0.005)$ but not different from that of $0.0 \mathrm{IC}(P=0.06)$ at $6 \mathrm{I}-\mathrm{I} 80$ minute range (D). At the range of $6 \mathrm{I}-180$ minutes during subjective night $(\mathrm{N}), 0.125 \mathrm{~T} / 0.0 \mathrm{IC}$-treated flies have lower sleep episodes than that of $0.125 \mathrm{~T}(P<0.005)$ but not different from that of $0.01 \mathrm{C}(P=0.2)$
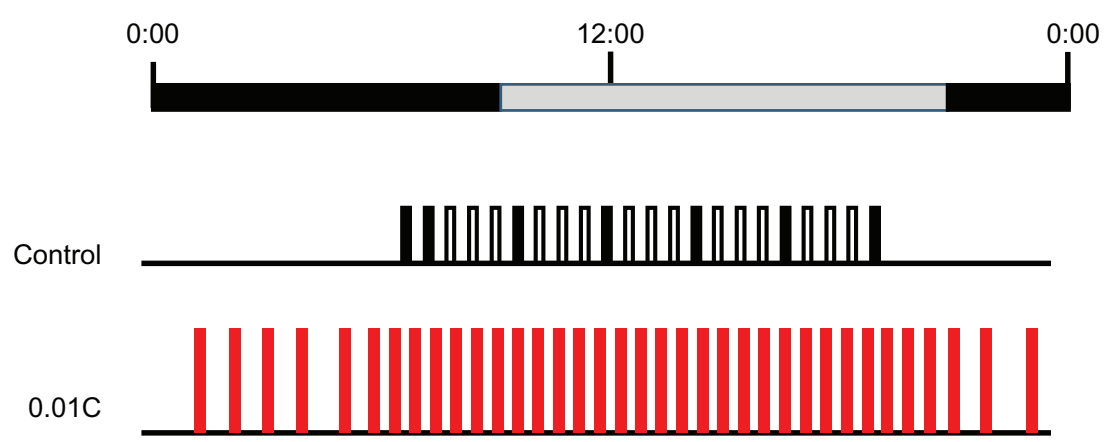

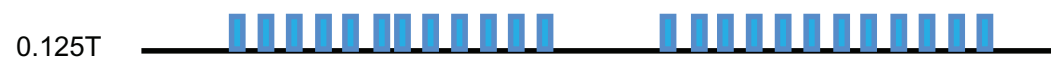

$0.125 \mathrm{~T} / 0.01 \mathrm{C}$

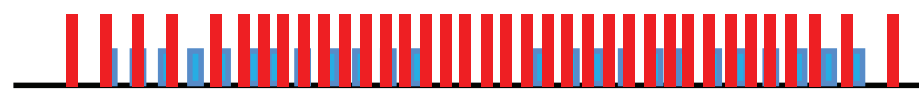

Figure S2 Schematic drawing of the actograms as seen in Figure 3B. The amplitude of locomotor activity is indicated by the height of the bar whereas sleep is shown as a space between the bars. Shaded rectangles represent subjective day (10:00 to 22:00), whereas solid black rectangles represent subjective night (22:00 to 10:00). Compared to the control, caffeine increases both amplitude and frequency of locomotor activity as well as reduces sleep. The locomotor activity in $0.125 \mathrm{~T}$ is lower and drifted to late night/early morning, accompanied by increase in sleep. When the two are combined $(0.125 \mathrm{~T} / 0.0 \mathrm{IC})$, total sleep is reduced more than that of caffeine or taurine alone.

Nature and Science of Sleep

\section{Publish your work in this journal}

Nature and Science of Sleep is an international, peer-reviewed, open access journal covering all aspects of sleep science and sleep medicine, including the neurophysiology and functions of sleep, the genetics of sleep, sleep and society, biological rhythms, dreaming, sleep disorders and therapy, and strategies to optimize healthy sleep. The journal welcomes

\section{Dovepress}

Submit your manuscript here: http://www.dovepress.com/nature-and-science-of-sleep-journal 